\title{
Electron-number statistics and shot-noise suppression by Coulomb correlation in nondegenerate ballistic transport
}

\author{
O. M. Bulashenko,* J. Mateos, D. Pardo, and T. González \\ Departamento de Física Aplicada, Universidad de Salamanca, Plaza de la Merced s/n, E-37008 Salamanca, Spain \\ L. Reggiani \\ Istituto Nazionale di Fisica della Materia, Dipartimento di Scienza dei Materiali, Università di Lecce, Via Arnesano, 73100 Lecce, Italy \\ J. M. Rubí \\ Departament de Física Fonamental, Universitat de Barcelona, Avenida Diagonal 647, E-08028 Barcelona, Spain
}

(Received 6 August 1997)

\begin{abstract}
Within a Monte Carlo simulation, we investigate the statistical properties of an electron flow injected with a Poissonian distribution and transmitted under ballistic regime in the presence of long-range Coulomb interaction. Electrons are shown to exhibit a motional squeezing which tends to space them more regularly rather than strictly at random, and to evidence a sub-Poissonian statistics with a substantially reduced Fano factor $F_{n} \ll 1$. The temporal (anti)correlation among carriers is demonstrated to be a collective effect which persists over the transit of several successive electrons, and results in a considerable (more than one order of magnitude) shot-noise suppression. [S0163-1829(98)03903-4]
\end{abstract}

The study of electronic noise in low-dimensional systems, and mesoscopic conductors in particular, has been receiving increasing attention in recent years. ${ }^{1-7}$ Indeed, by complementing the analysis of current-voltage characteristics, knowledge of statistical properties provides valuable information on the microscopic mechanisms ultimately responsible for charge transport. Uncorrelated carriers exhibiting Poissonian statistics are known to be characterized by a shotnoise power given by the Schottky formula $S_{I}=2 q I$ ( $q$ being the unit charge, and $I$ the average current). To a general extent the ideal $2 q I$ value can be reduced by imparting an anticorrelation to the electron stream, thus making the electron statistics become sub-Poissonian. This can be achieved by exploiting several mechanisms. Pauli correlation under degenerate conditions has been investigated in recent experiments $^{1-5}$ and theories (see detailed bibliography ${ }^{6}$ ). A $\frac{1}{2}$ noise-suppression factor has been found in a symmetric double-barrier junction. ${ }^{1-3}$ For a diffusive conductor a $\frac{1}{3}$ suppression has been demonstrated. ${ }^{7}$ The statistics of charge transmitted through a degenerate conductor has been found to be binomial, ${ }^{8}$ while that through a double-barrier junction is in between a Gaussian and a Poisson distribution. ${ }^{9}$

Another possibility to incorporate correlations among electrons is by means of long-range Coulomb repulsion. The desired effect can be achieved by virtue of a potential barrier near an injecting contact, which fluctuates synchronously with electron passages through it. In this way, an incoming Poissonian flow is converted into an outcoming subPoissonian flow. This effect is similar to that leading to shotnoise suppression in vacuum diodes, and discussed in the literature long ago, ${ }^{10}$ although, in the case of a device, the potential barrier should not necessarily be provided by space charge. In a semiconductor structure it might be, e.g., a constriction, as in the experiment on a quantum point-contact by Reznikov et al. ${ }^{4} \mathrm{We}$ note that, although the importance of Coulomb correlations on noise suppression in mesoscopic structures has been emphasized, ${ }^{11,12}$ only a few calculations are available, ${ }^{13,14}$ and the statistics of charge transmitted under Coulomb correlations has never been studied to the best of our knowledge.

It is the aim of the present Brief Report to address the problem of Coulomb correlation by investigating the carrier statistics under a nondegenerate ballistic transport regime through a Monte Carlo simulation. We have found the interesting result that just after the potential barrier, selfconsistently generated by the electron flow, the statistical variance of electron-number counts decreases with the distance from the barrier. Thus, correlated electrons exhibit $a$ motional electron-number squeezing, characterized by a reduced Fano factor $F_{n} \ll 1$.

For our analysis we consider the following simple model: a lightly-doped active region of a semiconductor sample sandwiched between two heavily doped contacts (of the same semiconductor) subject to an applied voltage bias $U$. The contacts are considered to be Ohmic (the voltage drop inside them is negligible), and they always remain at thermal equilibrium. Thus electrons are injected from the two opposite contacts according to a Maxwell-Boltzmann distribution at the lattice temperature $T_{L}$. To exclude correlations due to Fermi statistics, the electron gas is assumed to be nondegenerate. Electrons are injected with a Poissonian statistics, i.e., the time between two consecutive electron injections is generated with a probability per unit time $P(t)=\Gamma_{0} e^{-\Gamma_{0} t}$, where $\Gamma_{0}=\frac{1}{2} n_{c} v_{\text {th }} S$ is the injection-rate density, $n_{c}$ the carrier concentration at the contact, $S$ the cross-sectional area, $v_{\text {th }}$ $=\sqrt{2 k_{B} T_{L} / \pi m}$ the thermal velocity, and $m$ the electron effective mass. For the simulation we used the following set of parameters: $T_{L}=300 \mathrm{~K}, m=0.25 m_{0}\left(m_{0}\right.$ being the free electron mass), relative dielectric constant $\epsilon=11.7$, active region length $L=200 \mathrm{~nm}$, and $n_{c}=4 \times 10^{17} \mathrm{~cm}^{-3}$, much higher than the sample doping (here taken at $10^{11} \mathrm{~cm}^{-3}$, but the 


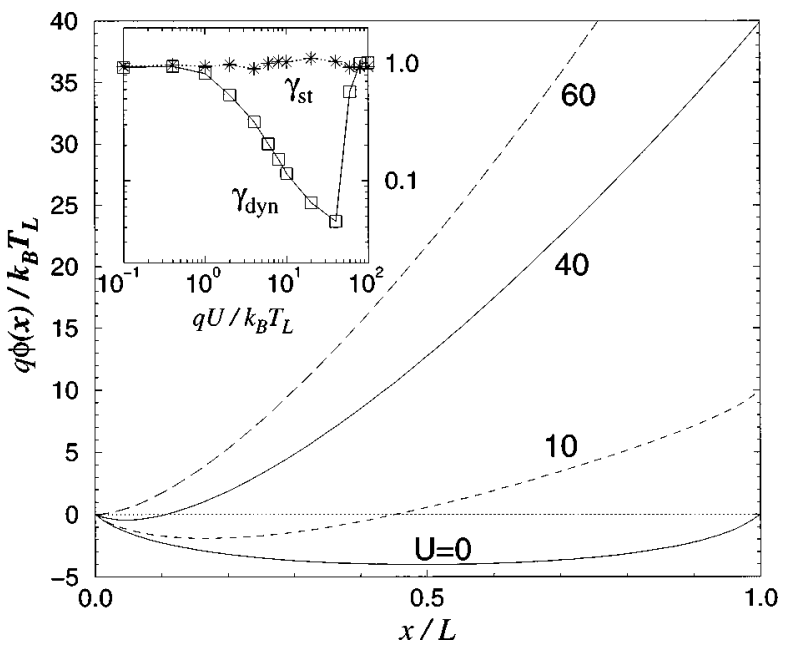

FIG. 1. Spatial profiles of the normalized potential $\phi(x)$ for different applied voltages $U$ (in units of $k_{B} T_{L} / q$ ). Inset: shot-noise suppression factor vs voltage $U$ for the dynamic PS $\left(\gamma_{\text {dyn }}\right)$ and static PS $\left(\gamma_{\mathrm{st}}\right)$, respectively.

same results are obtained up to $10^{15} \mathrm{~cm}^{-3}$ ). Under these conditions the electron dynamics is determined by the ratio $\lambda=L / L_{D c}$, where $L_{D c}$ is the Debye screening length associated with the electron injection density. The above set of parameters corresponds to $\lambda=30.9$.

We calculate the fluctuating current $I(t)$ and its lowfrequency fluctuation spectrum $S_{I}$ by an ensemble Monte Carlo simulator self-consistently coupled with a Poisson solver (PS). ${ }^{13}$ To illustrate the importance of Coulomb correlation, we provide the results for two different simulation schemes. The first one involves a dynamic PS: the fluctuations of the potential follow self-consistently the fluctuations of the space charge by virtue of the Poisson equation. The second scheme makes use of a static PS, which calculates the stationary potential profile only, so that carriers move in the frozen nonfluctuating electric field. Both schemes give exactly the same average current and steady-state spatial distributions of all first-order quantities, but they provide different noise levels and statistical distributions of transmitted carriers.

Figure 1 illustrates the potential minimum which, acting as a barrier for the electrons moving between the contacts, is shifted by changing the bias $U$. The corresponding noisesuppression factor $\gamma=S_{I} / 2 q I$ is reduced considerably when the dynamic PS is used, while for the static PS $\gamma \approx 1$ for all the biases (see the inset of Fig. 1). The noise suppression becomes particularly pronounced at $q U \approx 40 k_{B} T_{L}$, when the potential barrier almost vanishes completely, and its fluctuations affect the transmission of the most populated (lowvelocity) states of injected carriers. At the highest voltages the barrier disappears, and no shot-noise suppression is observed. Note that the shot-noise suppression factor by Coulomb interaction can achieve a value as low as desired in contrast to other mechanisms ( $\frac{1}{3}$ for diffusive conductors, $7 \frac{1}{2}$ for double-barrier junctions ${ }^{9}$ ) by increasing the ratio $\lambda$ $=L / L_{D c}$, i.e., by increasing the sample length and/or the carrier concentration at the contact, provided the transport remains ballistic. In the following we fix the bias at $U$ $=40 k_{B} T_{L} / q$, for which $\gamma \approx 0.045$.

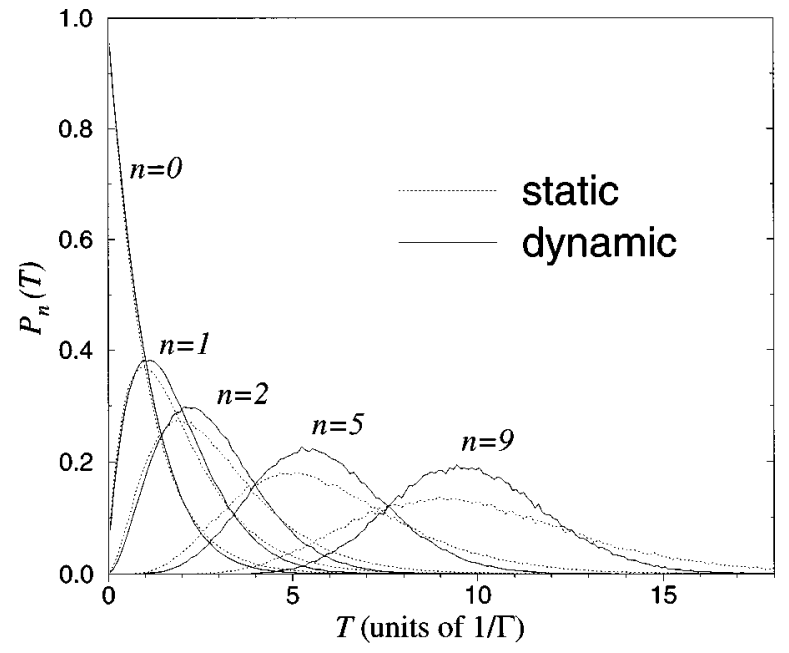

FIG. 2. Distribution function $P_{n}(T)$ of electron-counting statistics at the receiving contact for the static PS (dotted line, Poissonian statistics) and for the dynamic PS (solid line, sub-Poissonian statistics).

Having found the level of noise below the ideal Poissonian value, it is natural to ask which are the statistical properties of the transmitted carriers once they are temporally correlated. To address this question, we register the times of passage of electrons through different sections of the sample at a distance $x$ from the injecting contact. We register only those going from the cathode to the anode, since these are the only electrons contributing to the low-frequency current noise. With this procedure we are able to study how the carrier statistics imposed at $x=0$ (in our case Poissonian) modifies at different distances from the cathode. For each section $x$ the times of passage of carriers $\left\{t_{1}, t_{2}, \ldots, t_{i}, \ldots\right\}$ constitute a stationary stochastic point process, which can be described by specifying the random set $\left\{\tau_{i}\right\}$, where $\tau_{i}$ $=t_{i+1}-t_{i}$ is the time interval between events. By knowing the set $\left\{\tau_{i}\right\}$, we calculate the distribution function $P_{n}(T)$, which is the probability of detecting $n$ electrons during the observation time interval $T$. For a Poissonian process all time occurrences are statistically independent, which leads to the simple formula

$$
P_{n}(T)=\frac{(\Gamma T)^{n}}{n !} e^{-\Gamma T}, \quad n=0,1,2, \ldots,
$$

where $\Gamma$ is the rate density of events. Distribution (1) is characteristic of uncorrelated transport, and is tested to describe correctly the carrier statistics at the injecting contact at $x=0$. The distribution of carriers at the receiving contact depends crucially on the PS scheme (see Fig. 2). For the static PS, $P_{n}(T)$ is perfectly fitted by the same Poissonian formula (1) as for the injected carriers, but with a smaller value of the rate density $\Gamma=\kappa \Gamma_{0}$, with $\kappa \approx 0.65$. Its reduction is caused by the part of injected carriers, which is reflected by the potential barrier back to the contact. ${ }^{15}$ In contrast, for the dynamic PS the distribution function no longer obeys formula (1). Figure 2 shows the following differences between dynamic and static PS's: (i) for each $n \geqslant 1$ the maximum of the distribution is shifted to longer $T$; (ii) the probability distribution is narrowed; and (iii) the higher the index $n$, the more the dynamic case deviates from the static one, 


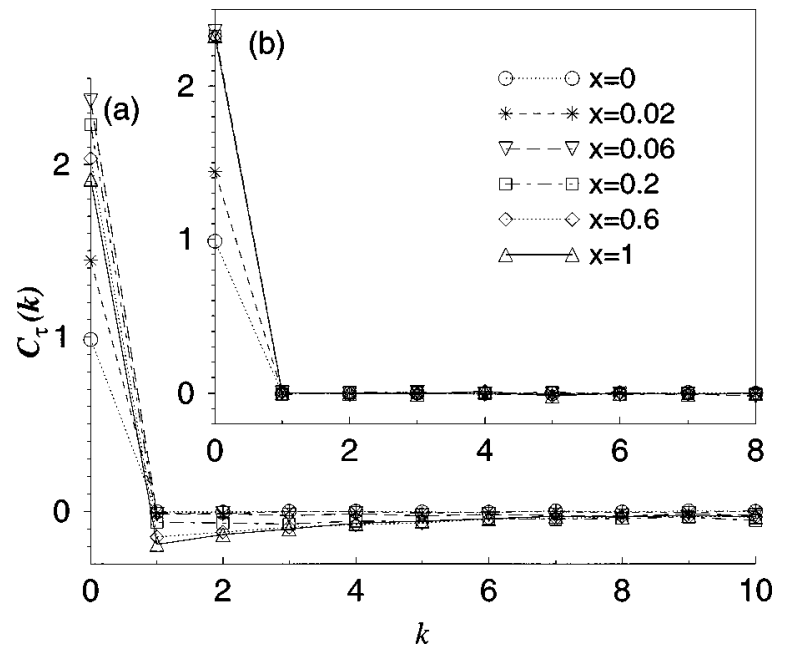

FIG. 3. Correlation function of interevent times as a function of the number of successive events at different distances $x$ from the injecting contact for the dynamic (a) and static (b) PS's, respectively. For a better view discrete points are connected by lines. $x$ is in units of the sample length $L$.

the distribution profiles becoming more symmetrical and closer to a Gaussian shape. We remark that the difference at point (ii) can be interpreted as a motional squeezing of electron number, and corresponds to a higher regularization of the carrier passage due to correlation among them.

The temporal correlation among carriers can be characterized by the autocorrelation function of the interevent times as a function of the number of successive events defined as

$$
C_{\tau}(k)=\left\langle\left(\tau_{j}-\bar{\tau}\right)\left(\tau_{j+k}-\bar{\tau}\right)\right\rangle, \quad k=0,1, \ldots .
$$

Here $\bar{\tau}$ is the mean of the random variable $\tau_{i}$, and angular brackets denote average over the reference index $j$. Figure 3 shows the function $C_{\tau}(k)$ calculated for static and dynamic PS's using the random sets $\left\{\tau_{i}\right\}$ registered at different positions $x$ along the sample. First, we note that for the static PS the correlation function is zero for all $k \neq 0$ [Fig. 3(b)]. This means that the absence of correlation among carriers imposed by the Poissonian injection is preserved at any section of the sample. Indeed, the electric potential being frozen, no additional correlation is introduced inside the system. The only nonzero value is the variance $C_{\tau}(0)=\operatorname{Var}(\tau)=\overline{\tau^{2}}$ $-\bar{\tau}^{2}$, which increases with $x$ until $x$ coincides with the minimum of the potential profile (here the carrier flow is decreased due to the reflection), and then saturates for larger values of $x$ (see Fig. 4). In contrast, for the dynamic PS $C_{\tau}(k)$ is in general nonzero for $k \neq 0$ and, moreover, it is negative by decaying to zero at increasing values of the index $k$ [Fig. 3(a)]. Negative values of $C_{\tau}(k)$ start to appear just after the potential minimum, their magnitude tending to increase for longer distances. The negative sign means that once a time interval $\tau_{m}$ longer than the average value $\bar{\tau}$ is registered, to compensate for such a deviation the successive $\tau_{m+1}$ is expected to be shorter than $\bar{\tau}$. The mechanism responsible for this compensating behavior is the selfconsistent redistribution of the potential which, by varying the barrier height, introduces a correlation into the carrier flow. From Fig. 3(a) we estimate that the number of consecu-

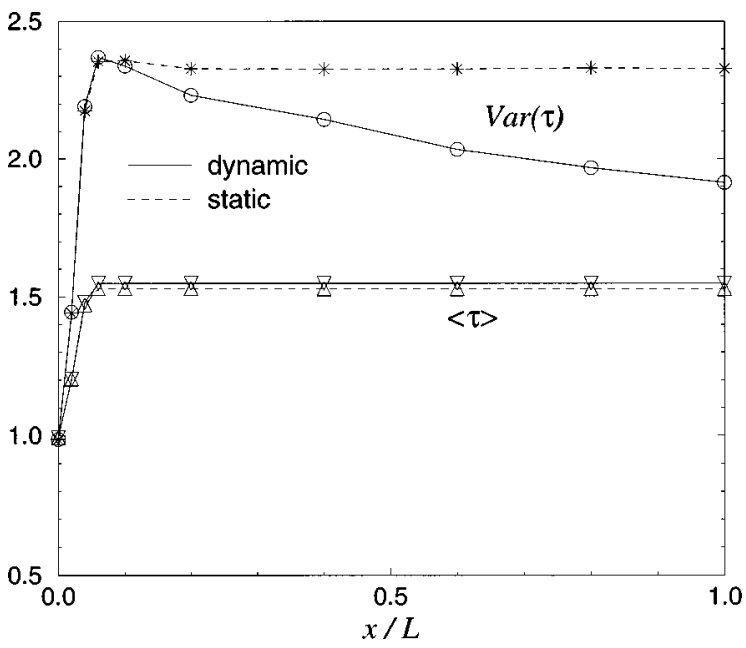

FIG. 4. Mean and variance of interevent times as a function of distance $x$ from the injecting contact. $\tau$ is multiplied by the injection rate $\Gamma_{0}$.

tive carrier passages over which the correlation persists is $\approx 6$ [i.e., $C_{\tau}(k) \approx 0$ for $k>6$ ]. It should be stressed that the mean time between consecutive electrons is not influenced by the long-range Coulomb correlation, since $\bar{\tau}$ is related to the average current. Figure 4 shows that, for both static and dynamic PS's, $\bar{\tau}$ increases in the region before the barrier $(x \leqslant 0.06 L)$, due to the loss of the part of injected electrons which are reflected by the potential barrier, and then saturates. The Coulomb correlation is mirrored by the second moment $\operatorname{Var}(\tau)$ which, for the dynamic PS, is found to decrease with distance just after the barrier (see Fig. 4).

The autocorrelation function $C_{\tau}(k)$ refers to the statistical distribution of interevent times $\left\{\tau_{i}\right\}$ which, in turn, is related to the operation mode of registering the carrier arrival times. Another operation mode is the number counting, for which the number of carriers $n_{i}^{T}$ in a given time interval $T$ is registered. For this mode the statistical information is carried by the random variable $\left\{n_{i}^{T}\right\}$ which depends on the observation time $T$ as a parameter. The first-order statistics of the random set $\left\{n_{i}^{T}\right\}$ is described by the distribution function $P_{n}(T)$ shown, for example, in Fig. 2 for the carriers at the receiving contact. A better insight into the carrier correlation can be obtained by calculating the correlation function

$$
C_{n}(k, T)=\left\langle\left(n_{j}^{T}-\bar{n}^{T}\right)\left(n_{j+k}^{T}-\bar{n}^{T}\right)\right\rangle, \quad k=0,1, \ldots,
$$

representing the second-order statistics. Here $\bar{n}^{T}$ is the mean number of counts during the observation time interval $T$. For fixed $T \sim \bar{\tau}, C_{n}(k, T)$ behaves like $C_{\tau}(k)$, i.e., it is negative for $k \geqslant 1$, with its lowest value at $k=1$ [like in Fig. 3(a)]. Therefore, $C_{n}(1, T)$ can be a good measure of the correlation between the number of counts. This quantity, normalized to the mean number of counts $\bar{n}^{T}$, is shown in Fig. 5 for the statistics registered at the receiving contact under dynamic regime. $C_{n}(1, T)$ is seen to display a minimum as a function of $T$ (indicating the maximum of correlation) for $T \approx 5 / \Gamma$. This means that the maximum of the correlation occurs when the observation time interval $T$ is chosen such that the number of counts is $\vec{n}^{T} \approx 5$. In fact, this is another estimation of 


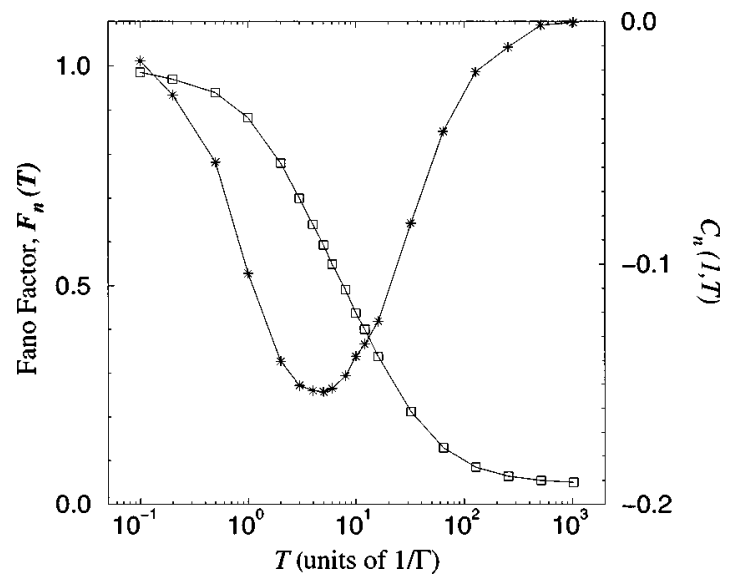

FIG. 5. Fano factor $F_{n}(T)$ (left axis, squares), and correlation function of the number of counts between two consecutive observation intervals, $C_{n}(1, T)$, normalized by $\bar{n}^{T}$ (right axis, stars), calculated at the output contact within the dynamic scheme.

the number of consecutive carriers over which the correlation persists, in addition to that obtained from the approximate decaying range of $C_{\tau}(k)$ of Fig. 3(a) $(k \approx 6)$.

The correlation function for $k=0$ gives the variance of the number of counts $C_{n}(0, T)=\operatorname{Var}\left(n^{T}\right)=\overline{\left(n^{T}\right)^{2}}-\left(\bar{n}^{T}\right)^{2}$. In Fig. 5 we also present the ratio of the variance to the mean $F_{n}(T)=\operatorname{Var}\left(n^{T}\right) / \bar{n}^{T}$, known as the Fano factor in photoncounting statistics. ${ }^{16}$ It characterizes the degree of deviation of the counting statistics from the Poissonian one, whenever $F_{n}(T) \neq 1$. We have found that, for the static PS, $F_{n}(T)=1$ always, while for the dynamic PS it decreases with $T$ (see
Fig. 5). Notice that if the observation time $T \ll 1 / \Gamma, F_{n}(T)$ $\approx 1$, i.e., Coulomb correlation among carriers cannot be detected by any means, and the observed statistics of carriers is always Poissonian. For $T \gg 1 / \Gamma$ the correlation between the counting intervals is lost $\left[C_{n}(1, T) \rightarrow 0\right]$, while the Fano factor approaches its minimum value at the shot-noise suppression factor $F_{n}(\infty) \equiv \gamma \approx 0.045$ (Fig. 5).

In conclusion, within a Monte Carlo scheme we have investigated the statistics of carriers which are Poissonian injected and transmitted through a nondegenerate ballistic structure in the presence of long-range Coulomb interaction. The sub-Poissonian statistics exhibited by carrier number fluctuations registered at the receiving contact provides an interesting example of motional squeezing, in analogy with photon satististics. The negative value of the correlation function of both the time intervals between consecutive electron passages and the number of counts in successive observation time intervals, mirrors the temporal correlation of transmitted carriers introduced by the presence of space charge. The small value of the Fano factor $\left(F_{n} \ll 1\right)$ found here, besides being of fundamental interest, offers interesting perspectives in communication systems. Indeed, a subPoissonian electron flux can generate a photon-numbersqueezed light which, in turn, can be used to enhance a channel capacity to transmit information in optical communication systems. ${ }^{16}$

This work was supported by the Comisión Interministerial de Ciencia y Tecnología through the Project No. TIC950652, by the DGICYT of the Spanish Government under Grant No. PB95-0881, and Ministero dell' Università e della Ricerca Scientifica e Tecnologica (MURST).
*Present address: Dept. Física Fonam., Univ. Barcelona, Diagonal 647, 08028 Barcelona, Spain. Electronic address: oleg@ffn.ub.es

${ }^{1}$ Y. P. Li et al., Phys. Rev. B 41, 8388 (1990).

${ }^{2}$ H. C. Liu et al. Phys. Rev. B 51, 5116 (1995).

${ }^{3}$ H. Birk et al., Phys. Rev. Lett. 75, 1610 (1995).

${ }^{4}$ M. Reznikov et al., Phys. Rev. Lett. 75, 3340 (1995).

${ }^{5}$ A. Kumar et al., Phys. Rev. Lett. 76, 2778 (1996).

${ }^{6}$ M. J. M. de Jong and C. W. J. Beenakker, in Mesoscopic Electron Transport, edited by L. P. Kowenhoven, G. Schön, and L. L. Sohn (Kluwer, Dordrecht, 1997).

${ }^{7}$ C. W. J. Beenakker and M. Büttiker, Phys. Rev. B 46, 1889 (1992); K. E. Nagaev, Phys. Lett. A 169, 103 (1992); A. H. Steinbach, J. M. Martinis, and M. H. Devoret, Phys. Rev. Lett. 76, 3806 (1996).

${ }^{8}$ L. S. Levitov and G. B. Lesovik, Pis'ma Zh. Eksp. Teor. Fiz. 58, 225 (1993) [JETP Lett 58, 230 (1993)]; L. S. Levitov, H. Lee, and G. B. Lesovik, J. Math. Phys. 37, 4845 (1996).

${ }^{9}$ M. J. M. de Jong, Phys. Rev. B 54, 8144 (1996).

${ }^{10}$ A. van der Ziel, Noise (Prentice-Hall, Englewood Cliffs, NJ, 1954), Chaps. 5-1 and 14-2.

${ }^{11}$ R. Landauer, Phys. Rev. B 47, 16427 (1993); Physica B 227, 156 (1996).

${ }^{12} \mathrm{M}$. Büttiker, in 14th International Conference on Noise in Physical Systems and 1/f Fluctuations, edited by V. Bareikis and R. Katilius (World Scientific, Singapore, 1995), p. 35.

${ }^{13}$ T. González, O. M. Bulashenko, J. Mateos, D. Pardo, and L. Reggiani, Phys. Rev. B 56, 6424 (1997).

${ }^{14}$ T. González, O. M. Bulashenko, J. Mateos, D. Pardo, L. Reggiani, and J. M. Rubí, Semicond. Sci. Technol. 12, 1053 (1997).

${ }^{15}$ In fact, the current for the present applied voltage is $I=\kappa I_{s}$, with $I_{s}=q \Gamma_{0}$ the saturation current.

${ }^{16}$ B. E. A. Saleh and M. C. Teich, Proc. IEEE 80, 451 (1992). 